3.

\title{
Fall von Abreissung des Kindkopfes während der Geburt.
}

\author{
Mitgetheilt von Dr. E. Strauss, \\ Kreisarzt zu Talsen in Curland.
}

Vor mehreren Jahren kam in meinem Wirkungskreise, auf einem entiegenen Bauernhofe der eben so seltene als entsetzliche Fall vor, dass eine "sogenannte“ Hebeamme (denn bier in Curland ist fast jedes aite Wejb eine Hebearnme) einem mit den Füssen vorankommenden lebenden Kinde den Kopf vom Rumpfe abgerissen batte. Statt nun aber nach all dem Unglück sich blos passiv zu verhalten und die Ankunft des Arztes abzuwarten, hatte sie noch recht klug zu handeln geglaubt und die Zwischenzeit dazı benutzt, den Kopf wo möglich mit der Hand zu fassen, um ihn aus dem Becken zu zieben. Durch diese Manipulationen hatte sie aber nicht allein die letzten lïäfte der Gebärenden erschöpft, sondern, was noch viel schlimmer war, nach gänzlichem Aufhören der Wehen den Kopf wieder hoch in das grosse Becken hinaufgeschoben und dadurch eine Metrorrhagie veranlasst, welche mich die Patientin schon fast pulslos und sterbend antreffen liess. Mit der Zange den Kopf zu fassen, war bei dem hohen Stande desselben und seiner Beweglichkeit unmöglich. Ich musste die ganze Hand einführen, um mich von der Lage desselben zu überzengen; er war in dem grossen Becken so gestell, dass seine Längenaxe dem Querdurchmesser des grossen Beckens entsprach, die Pfeilnaht sah dem Beckenausgange zu, die kleine Fontanielle nach rechts, Die Hebeamme hatte den Kopf mithin ganz um seinen Längendurcbmesser rotirt, so dass das Foramen occipitale jetzt nach der Leber der Mutter hin gerichtet war. In meiner Rathlosigkeit und bei dem absoluten Mangel an Wehen, der Gefahr im Verzuge - dachte ich anfangs daran, das Foramen occip. wieder vor den Beckenausgang zu drehen, den stumpfen Haken in dasselbe einzufübren und so den Kopf zu extrahiren. Da fielen mir in der rechten Seite des Beckens ein Paar Stränge zwischen die Finger. Der eine erwies sich als die abgerissene Nabelschnur, den anderen aber, der mit einer Menge kurzer Fortsätze versehen war, vermochte ich mir nicht früher zu deulen, als bis ich den Rumpf des Kinderleichnams genau untersucht. Der Hals war jedenfalls sehr nałe dem Kople - wie es sich nachher ergab, zwischen Atlas und Epistropheus - abgerissen, die Haut des Halses fast zirkelrund, von Muskeln schien am Haise bichts zu fehlen, dagegen war der Wirbelkanal, so weit ich sehen und mit einer Stricknadel untersuchen konnte, leer. Jener zweifelhafte Strang, der nach einer Rotation des Kopfes in die Vagina hinabglitt, war also offenbar nichts anderes als das am Kopf zurückgebliebene Rückenmark in seinen Häuten. Dafür sprachen auch die kleinen einander gegenüberstehenden kürzeren und längeren Fortsätze. Ich zog an dem Strange, und der Kopf folgte auch dem Zuge so weit, dass es mir endlich möglich wurde, die Zange 
an denselben anzulegen und ihn zu extrabiren. Trotz aller angewandten Mittel traten indess keine Wehen mehr ein, die Hämorrhagie dauerte fort und die Wöchnerin starb nach einigen Stunden.

So hatte denn die biedere "Hebeamme" erst mit übermenschlicher Anstrengung ein Kinderleben im eigentlichen Sinne des Wortes zerrissen und darauf noch mit klugem Eifer die Mutter zum Verbluten gebracht. Nachher in dieser Beziehung angestelle Versuche haben erwiesen, dass wirklich eine ausserordentliche hraft dazu gehort, dem Leichnam eines neugebornen Kindes den Kopf vom Rumpfe zu reissen. Nachdem ich mich in gelegentlichen Fällen öfter vergeblich bemüht batte, den Kopf von neugebornen Kinderleichen durch Ziehen vom Rumpfe zu trennen und es mir selbst bei der grössten Kraftanstrengung und Ausdauer nicht möglich geworden war, den Kopf zum weichen zu bringen, trieb mich die Neugierde dazu, den betreffenden Versuch mit Hü]fe von Gewichten zu wiederholen, um die Kraft zu messen, die erforderlich ist, um den Kopf eines Neugebornen vom Rumpfe zu reissen.

Fünf Kinderleichen habe ich dazu benutzt und Folgendes ermittelt.

No. 1. Leichnam eines neugebornen ausgetragenen Knaben von $6 \frac{1}{2}$ Pfund Gewicht und gutem Ernährungszustand. Das Kind batte nach der Geburt nicht gelebt. Ein Handtuch ward um die Schultern des Leichnams geschlungen und an einer Leitersprosse befestigt, ein anderes Handtuch, welches so um den Kopf geschlungen war, dass seine beiden Enden seitlich an den Ohren vorüber zur kleinen Fontanelle verliefen, ward mit der Waagschale in Verbindung gebracht. 9 Centner 14 Pfund waren erforderlich, um unter allmäliger Belastung der Waagschale nach einer Einwirkung von 12 Minuten Dauer den Kopf vom Rumpfe zu trennen.

No. 2. Leichnam eines neuzebornen ausgetragenen Mädchens von etwas über 7 Pfund Gewicht und gutem Ernäbrungszustand. Keine Spur von Fäuniss. Nach der eben beschriebenen Methode waren 10 Centner 11 Pfund Belastung bei einer Daver von 8 Minuten nöthig, um den Kopf znm weichen zu bringen.

No, 3. Etwas verwester Leichnam eines neugebornen ausgetragenen Mädchens yon $7 \frac{1}{2}$ Pfund Gewicht, das nach der Geburt nicht gelebt hatte. Es waren 10 Centner 4 Pfund Belastung bei einer Dauer von 18 Minuten nöthig.

No. 4. Neugeborner Knabe von $7 \frac{1}{2}$ Pfund Gewicht und gutem Ernährungszustand - ausgetragen -- hat gelebt. Leichnam frisch. 11 Centner 14 Pfund nöthig bei einer Einwirkung von 12 Minuten Dauer.

No. 5. Neugebornes Mädchen von 7 Pfund Gewicht, schlecht ernährt. Hautwunde am Halse. Der Leichnam hat 11 Tage auf einem Sumpf gelegen. 7 Centner 15 Pfund nöthig bei einer Einwirkung von 6 Minuten Daner.

Aus vorstehenden Experimenten ergiht sich, dass im Durchschnit mindestens eine Kraft von 10 Centner erforderlich ist, um einem neugebornen Kinde bei ununterbrochener Einwirkung des Gewichts während einer Daver ron 10 Minuten den Kopf vom Rumpfe zu reissen.

Von Interesse ist mir ferner die Wabrnehmung gewesen, dass in der Mehrzabl der Fälle, ganz wie in jener oben erzählten Zerreissung eines lebenden Kindes - das Rückenmark sammt seinen Häuten in seiner ganzen Länge am Kopfe 
hängen geblieben und die Zerreissung der Wirbelsäule selbst zwisehen dem Atlas und Epistropheus zu Stande gekommen war, so zwar, dass der Processus odontoideus sich von dem Epistropheus gelöst hatte und an seinen starken Bändern im Ringe des Atlas zurückgeblieben war, welcher letztere Umstand ganz besonders die Annahme zu bestätigen scheint, dass der Proc. odontoideus im embryonalen Zustande vielmehr dem Atlas als dem Epistropheus angehöre.

4.

Zahlreiche Erkrankungen durch gährendes Bier.

\author{
Beobachtet von Dr. E. Strauss.
}

Auf dem bel Talsen belegenen Gute P ... war das vom Gutsherrn seiner Bauernschaft alljährlicb gegebene Volksfest gefeiert worden. Am Tage nach diesem Feste latte ich auf dem genannten Gute und den umliegenden Beihöfen und Gesinden über 70 Patienten zu besuchen, die alle von einer und derselben Krankheit befallen waren. Alle fieberten lebhaft, batten zahlreiche Durchfälle, fortwährendes Erbrechen und starke Schweisse, bei vielen grosse Prostration der Rräfte und comatöser Zustand. Am zweiten Tage waren alle wieder gesund.

Sofort angestellte genaue Nachforschungen ergaben das sichere Resultat, dass nur solche Personen erkrankt waren, die das auf dem Volksfeste reichlich vertheilte Bier getrunken batten, und zwar stand die Intensital der Erkrankung im geraden Verhältniss zu der Quantität des von ihnen genossenen Bieres. An sich schädliche Stoffe waren, wie es die Untersuchung mit Wahrscheinlichkeit ergab, dem Biere nicht beigemischt worden, wohl aber hatte der Hofesbrauer, da er die erforderliche Quantität ausgegobrenen Bieres nicht vorräthig gebabt, ein ganz frisches Gebräu zum Volksfeste geliefert, dem er, nach seinem eigenen Eingeständniss, am Tage vor dem Volksfeste eine grössere Quantität Hefe zugesetzt hatte. Die überaus intensiven Krankheitserscheinungen bei den 70 Patienten müssen also auf den Gährungsprozess bezogen werden, der, statt im Fasse, in den Leibern der Erkrankten vor sich gegangen war, und bönnten mithin als eine Vergiftung durch Koblensäure angesehen werden.

Talsen, im Februar 1864. 